\title{
Isolation of Soil Mycoflora from Agricultural Fields of Narmada District
}

\author{
Alpesh Bhimani ${ }^{1 *}$ and Nikunj Sohaliya ${ }^{2}$ \\ *1Department of Agricultural Microbiology, Navsari Agricultural University, Navsari. ${ }^{2}$ Department of Plant Pathology, Navsari \\ Agricultural University, Navsari
}

\begin{abstract}
The soil samples were collected from different fields of Narmada District. Soil samples were collected in two different zones i.e. rhizoplane and rhizosphere. Then collected samples were inoculated on Potato Dextrose Agar (PDA) medium which was supplemented by suitable antibiotics such as Chloramphenicol by adopting soil dilution method and soil plate method. The present study was conducted to find out the fungal diversity in agricultural fields in Narmada. A total of 154 colonies were isolated. From this investigation, 14 species of fungi belongs to 6 different genera were isolated and identified whereas 23 strains left unknown. Identification and characterization of the soil mycoflora were done with the help of authentic manuals of soil fungi.
\end{abstract}

Key Words: Micro Fungi, Culture Media, Isolation, Fungal Diversity, Narmada.

\section{INTRODUCTION}

Soil contain large amount of microbial diversity which can interact with the plants resulting in useful effect or in harmful consequences. Among all microbes fungi are an important component of the soil microbiota (1) and they are present as mycelia fragments, rhizomorphs or as spores. They are playing significant role in soil and plant nutrition. Some fungi are saprophytic means they lives on dead and decaying organic matter, so breaking it down and converting to available forms to higher plants by excreting a wide range of degraded enzymes that attack virtually any organic material. These types of degrading activities make fungi important participants in recycling natural/agricultural waste in our environment. But sometimes unfortunately their degrading activity also results in the undesired growth of fungi that degrade useful materials (2). Fungi grow on diverse habitats in nature and are cosmopolitan in nature. In laboratory condition they can isolated on specific culture medium for cultivation, preservation, microscopic examinations and different biochemical and physiological characterization. The diversity richness of a microbial community and relative abundance of individual species have been considered as measures of functional activities of the group in the particular habitat (3).

Micro-organisms like fungi, bacteria, actinomycetes and other organisms colonize in different type of habitats and different substrates. Besides producing diseases, they play important role in plant health and productivity. Two important terms viz., Rhizosphere and Rhizoplane are necessary to consider while learning with soil microorganisms. The Rhizosphere is a microecological zone in direct proximity of plant roots. It is functionally defined as the particulate matter and microorganisms that cling to roots after being gently shaken in water. The rhizosphere is a metabolically busier, faster moving, more competitive environment than the surrounding soil while The Rhizoplane is the region around the root epidermis and outer cortex where soil particles, bacterial structures and fungal hyphae adhere. There are more microbes in the rhizoplane than in the rhizosphere. The diversity of the microbial population is determined by counting the number of colony forming units (CFUs). By spreading the extracted soil

*Address of Correspondence: Bhimani Alpesh, Dept. of Agricultural Microbiology, Navsari Agricultural University, Navsari - 396421, Gujrat, India. E-mail- bhimani4@gmail.com

(Received 18 January 2021; 20 January 2021; Accepted 28 January 2021) 
microorganisms across agar and counting the number of individual group of microorganisms, the CFUs can be determined. Endophytic fungi and bacteria means which lives within the cells of the roots are not considered a part of the rhizoplane.

\section{MATERIALS AND METHODS}

Soil sample were collected from different location of Narmada district in Gujarat, India. Potato Dextrose Agar (PDA) medium was used for isolation of different groups of fungi which influence the vegetative growth and colony morphology. (PDA was prepared by using extract from $250 \mathrm{~g}$ of potato boiled and filtered, dextrose $20 \mathrm{~g}$, agar $15 \mathrm{~g}$ and distilled water $1000 \mathrm{ml}$ ) (2). The $\mathrm{pH}$ was maintained at 5.5-6 as it is optimum for the growth and sporulation of majority of fungi.

Soil sample were collected from a depth of $15 \mathrm{~cm}$ with the help of a sterilized cork borer as majority of fungi are microscopic and shows vast variation in quantitatively and qualitatively aspects with change in sites of collection and at different depths. The collected soil was emptied into sterilized polyethylene bags. Each sample bag was labeled appropriately by indicating the site of collection, time, date and place of collection. Then samples were taken to the laboratory using sterilized cellophane bags (2).

\section{Isolation of fungi from the soil samples}

The soil dilution (4) and soil plate method (5) on media like Potato Dextrose Agar (PDA) used as isolation techniques.

1. Soil Dilution Plate Method (4): soil dilutions were made by adding $1 \mathrm{~g}$ of soil from each sample in $10 \mathrm{ml}$ of sterile distilled water. Dilutions of $10^{-3}, 10^{-4}$ and $10^{-5}$ were used to isolate fungi to avoid over population of the fungal colonies. $1 \mathrm{ml}$ of the suspension from each concentration was added to sterile Petri dishes with three replication of each dilution, containing sterilized Potato Dextrose Agar medium. Chloramphenicol was added to the medium for preventing bacterial growth, before pouring into Petri plates. The plates were then incubated at $27 \pm 2{ }^{\circ} \mathrm{C}$ for $4-7$ days. Micro-organisms were easily isolated because they have formed surface colonies that were well dispersed.

\section{Soil Plate Method (5):}

About $0.005 \mathrm{~g}$ of soil sample was scattered on the bottom of sterile Petri dish and molten cooled $\left(40-45{ }^{\circ} \mathrm{C}\right)$ agar medium PDA was added. Then it was rotated gently to disperse the soil particles evenly in the agar medium. Then plates were incubated at $27 \pm 2{ }^{\circ} \mathrm{C}$ for 4 5 days. One isolate of each fungal genus from each soil sample were selected by random for further sub-culturing and experiments. The pure cultures were maintained on Potato Dextrose Agar Slants.

\section{Identification of the Fungi}

Normally identification of the fungal species can be carried out by morphological characteristics of the colony and microscopic examinations (6). The colony growth like length and width of the colony, the presence or absence of aerial mycelium, the color and other pigment production were the macro morphological characters evaluated. Although molecular methods continue to improve and become more rapidly available, microscopic observation and cultural character remain commonly used and important tools for identification of fungal species (6) .The fungi were identified with the help of standard procedure and relevant literature $(7,8)$. Inoculating needle was flamed over the burning burner. Then using that needle, a small portion of the growth from the culture plate was transferred into the drop of lactophenol in cotton blue on the slide. The specimen was teased carefully using needle to avoid squashing and over-crowding of the mycelium (2). The specimens were observed under the microscope for microscopic identification.

\section{Statistical Analysis}

The population density was expressed in terms of Colony Forming Unit (CFU) per gram of soil with dilution factors. The percent contribution of each isolate was calculated by

$$
\% \text { Contribution }=\frac{\text { Total No. of CFU of an individual Species }}{\text { Total No. of CFU of all sps }} \times 100
$$

Where, CFU - Colony Forming Unit 


\section{RESULTS AND DISCUSSION}

Soil micro-organisms act as essential determinants of plant community variety and productivity (9). Different environmental factors such as the soil $\mathrm{pH}$, moisture, temperature, organic carbon and nitrogen play an important role in the distribution of mycoflora. The soil mycoflora in four different village of Narmada districts were observed. Soil dilution plate and soil plate methods were used for the isolation of fungi during this study. Total number of fungal colonies isolated on Petri plates containing PDA medium were 154. As mentioned earlier, soil dilution plate (10) and soil plate method (5) were used for the isolation of fungi during this study. A large number of species and colonies were isolated on soil plates than on dilution plates and further the total number of species isolated decreased with increased dilutions of the samples. The purification of the culture was carried out by using culturing of the hyphal tips and then transferred to fresh agar slants of PDA medium.

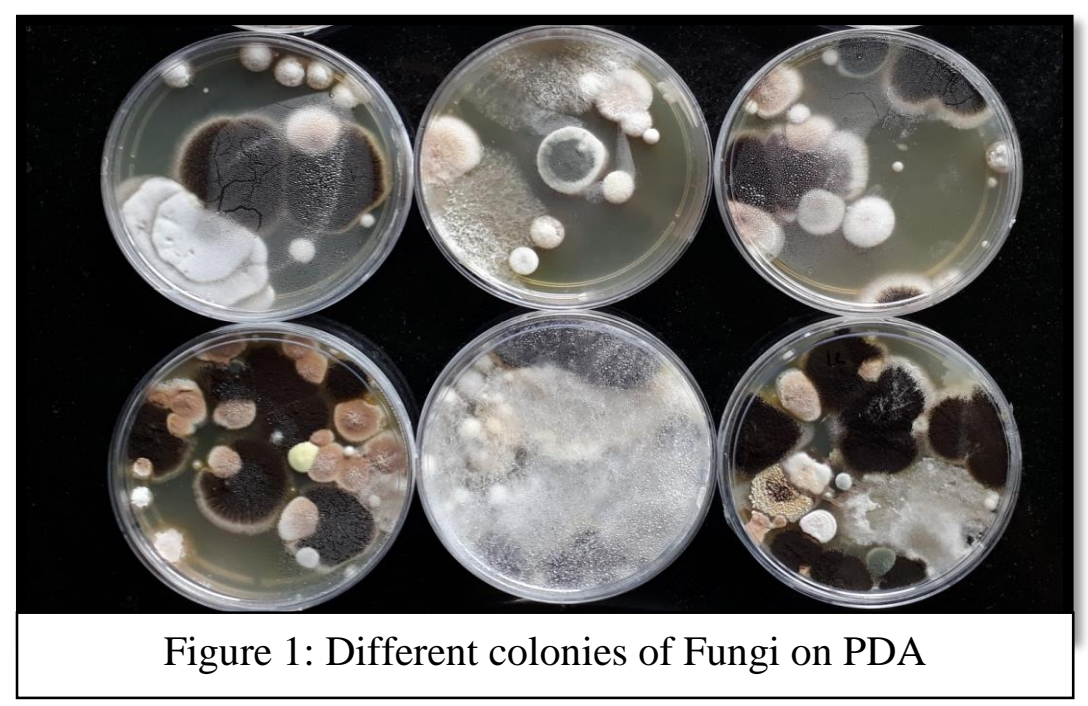

These fungi are the major decomposers of dead organic waste and have important role in recycling of nutrients in natural and modified ecosystems (11). All four soil samples from four different villages were examined for fungal diversity. The experiment resulted in presence of 14 species of fungi were identified and characterized. The maximum fungal species belonged to Deuteromycotina (127 species) and Zygomycotina (4 species) and 23 colonies were left unknown on the plates containing PDA medium. Due to its simple formulation and efficient to support wide range of fungal growth, PDA medium is the most commonly used culture medium and it was stated to be the best media for mycelia growth by several workers $(12,13)$. Characterization of the isolates were up to genus level and to the species level was made by using the macro-morphological and micromorphological characters by use of authentic manuals of soil fungi.

Our findings were similar to those isolated by Rasheed et al. (14). Aspergillus species particularly like A.flavus and A.niger, Penicillium and Rhizopus were isolated only from the soil whereas Trichoderma species,
Alternaria alternata, Curvularia lunata and Fusarium species were obtained from both soil and plant parts. In our study, among the obtained fungal isolates the genera Aspergillus and Penicillium were dominant on media used. The most common isolates among them viz., $A$. clavatus, A. flavus, A. fumigatus, A. nidulans, A.niger, A. restrictus, $A$. terreus, Curvularia clavata, C. lunata, Fusarium oxysporium, F. solani, Penicillium Chrysogenum, $P$. frequentens, $P$. funiculosum, Rhizopus stolonifer, Trichoderma harzianum, T.viride, T. virens, T. longibracheatum were isolated and characterized. The percent contributions of different soil mycoflora of all four villages were evaluated.

\section{CONCLUSION}

In the present experiment the soil sample of four different villages of Narmada districts viz; Sagai, Mohbi, Mal and Samot were studied for detection of the fungal diversity. Among the isolates Aspergillus and Penicillium were dominant in all agricultural fields of all areas mentioned due to high sporulation and production of bacterial antibiotics from the Penicillium species and production of different types of toxic materials from the Aspergillus 
species may prevent the growth of other fungal species. Trichoderma species were also isolated from these soil samples which show organic richness of soil. This study was conducted as a effort to understand the soil microbial diversity in the agricultural fields of Narmada district as soil microflora not only plays an important role in decomposition and contribute to biogeochemical cycling but also are responsible for the prevalence of diseases in the crop fields and availability of some minerals and nutrients.

\section{CONFLICT OF INTEREST}

There is no conflict of interest in this present research paper. This research work is not a part of any other studies and it is our original work.

\section{REFERENCES}

1. Ainsworth GC and Bisby GR. Dictionary of the fungi. Commonwealth Mycological Institute. 1995; 445.

2. Aina VO, Adewuni AAJ, Hauwa Haruna and Amina Zaraki. Isolation and identification of fungi associated with the deterioration of painted wall surfaces within Kaduna polytechnic. Asian Journal of Medical Sciences. 2011; 3: 250-253.

3. Kjoller A, Struwe S. Microfungi in Ecosystems.,fungal occurrence and activity in litter and soil. Oikos. 1982; 39: 389-422.

4. Waksman SA. A method for counting the number of fungi in the soil. J Bact. 1922; 7: 339341.
5. Warcup JH. The soil plate method for isolation of fungi from soil. Nature. Lond. 1950; 166: 117-118.

6. Diba K, kordacheh $\mathrm{P}$, Mirhendi SM, Rezaie S, Mahmoudi M. Identification of Asergillus species using morphological characteristics. Pal J Med sci. 2007; 23: 867-872. 7. Gilman JC. A Manual of soil fungi, 2nd Indian Edition, Biotech Books, Delhi, 2001.

8. Nagamani A, Kumar IK and Manoharachary C. Hand Book of Soil Fungi, I.K. International Publishing House Pvt Ltd, New Delhi, India. 2006.

9. Wardle DA, Bardgett RD, Klironomos JN, Setala H, Van der Putten WH, Wall DH. Ecological linkages between above ground and below ground biota. Science. 2004, 304: 16291633.

10. Waksman SA. Principles of Soil Microbiology. Williams and Wilkins Co. Baltimore, Md. 1927.

11. Gadd GM. Mycotransformation of organic and inorganic substrates. Mycologist. 2004; 18: 60-70.

12. Maheshwari SK, Singh DV, Sahu AK. Effect of several nutrient media, $\mathrm{pH}$ and carbon sources on growth and sporulation of Alternaria alternate. J Mycopathol Res. 1999; 37: 21- 23.

13. Saha A, Mandal P, Dasgupta S, Saha D. Influence of Culture Media and Environmental factors on mycelia growth and sporulation of Lasiodiplodia theobromae (Pat.). Griffon and Maubl. J Environ Biol. 2008; 29: 407-410.

14. Rasheed S, Dawar S, Ghaffar A. Location of fungi in groundnut seed. Pak J Bot. 2004; 36: 663-668. 